\title{
Outcome of ICSI cycles using pentoxifylline or theophylline to identify viable spermatozoa in patients with all immotile spermatozoa
}

\begin{abstract}
Introduction: Pentoxifylline and theophylline are used to identify viable sperm for ICSI in patients with all immotile spermatozoa. Although pentoxifylline is an effective tool, its use is controversial due to its possible detrimental effects on oocytes and embryos. Theophylline is another similar compound that is efficient in stimulating immotile spermatozoa. The objective of this study was to compare outcome of ICSI cycles after use of these 2 compounds.
\end{abstract}

Materials and Methods: Out of 112 patients, processed semen samples from 34 patients having all immotile sperm were treated with pentoxifylline and 78 samples with theophylline to identify viable sperm for ICSI. The sources of sperm were; 8 normal ejaculates, 2 TESE, 64 Micro-TESE, 11 MESA and 27 TESA samples. The Mean \pm SD ages were $38.9 \pm 10.7 \mathrm{yrs}$ and $31.7 \pm 6.2 \mathrm{yrs}$ for men and women, respectively. Ovarian stimulation, oocyte retrieval, sperm processing, embryo culture and embryo transfer were performed by standard protocols. Pentoxifylline and theophylline were added to ICSI drops. Oocytes were injected with motile or shaking sperm whenever available. Parameters were calculated as Mean $\pm \mathrm{SD}$ and percentages.

Results: Ages of men and women, number of eggs injected or fertilized, number transferred, biochemical pregnancies and clinical pregnancies were similar in both groups. Pentoxifylline was effective in $88 \%$ and theophylline in $90 \%$ cases. The miscarriage rate of $9 \%$ in pentoxifylline treated cases was significantly higher than $1.2 \%$ in theophylline treated cases. So far 11 deliveries of normal and healthy babies have been reported; 2 from pentoxifylline and 9 from theophylline.

Conclusion: These results indicate that theophylline is safe in identifying viable sperm for ICSI in cases with all non-motile sperm in fresh or frozen semen samples or those retrieved by surgical procedure.

Keywords: Pentoxifylline, Theophylline, Sperm activation, Immotile sperm, Viable sperm, ICSI, Micro-TESE
Volume 8 Issue I - 2017

\author{
Murid Javed, Othman Abdulrazak, Fatima \\ Abdelraouf, Sahar Bengawi, Tagwa Saad, \\ Abdulla Ahammed, Bilal Mahouk, Hamoud \\ Matrafi, Suleiman Najashi, Hamad Sufyan \\ Thuriah Medical Center, Saudi Arabia
}

Correspondence: Murid Javed, Thuriah Medical Center, PO Box 50246, Riyadh, I I 523, Saudi Arabia,Tel 96654 914-6434, Email murid.javed@thuriah.com.sa

Received: August 08, 2017 | Published: October 03, 2017
Abbreviations: ICSI, Intracytoplasmic Sperm Injection; TESE, Testicular Sperm Extraction; Micro-TESE, Microsurgical Sperm Extraction; MESA, Microsurgical Epididymal Sperm Aspiration; TESA, Testicular Sperm Aspiration; HOS, Hypo-Osmotic Swelling

\section{Introduction}

Sperm motility is an important parameter for success in intracytoplasmic sperm injection (ICSI) treatment. The sperm motility is due to its tail. If the sperm is not moving, it could be dead or alive. Normal live births have been obtained after ICSI with immotile sperm. ${ }^{1,2}$ Various procedures have been applied to pick viable sperm for ICSI including, hypo-osmotic swelling (HOS) test, ${ }^{3}$ addition of pentoxifylline, ${ }^{4}$ theophylline ${ }^{5}$ or papaverine,${ }^{6}$ laser-assisted selection, sperm tail flexibility test ${ }^{7-9}$ and platelet activation factor. ${ }^{10}$ The choice of method depends on type of sperm sample, availability of the material or equipment and preference of the embryology laboratory. Regardless of which method is employed, it results in higher fertilization rates and higher pregnancy rates in the difficult patient groups with asthenozoospermia or immotile testicular spermatozoa. ${ }^{7}$

Pentoxifylline has been used for detection of viable sperm before ICSI, ${ }^{2}$ for sperm cryopreservation to improve post-thaw motility. ${ }^{11}$
However, its effect on sperm, oocyte, embryo or new born are controversial. ${ }^{2}$ It increases sperm DNA damage. ${ }^{10}$ The DNA damage significantly increased with application of pentoxifylline compared to platelet activation factor. Further, despite improvement in motility, corresponding clinical pregnancy rates were similar between samples where pentoxifylline was added and non-treated controls. ${ }^{7}$ Theophylline, has been reported to be more reliable in stimulating testicular spermatozoa after thawing. Its immediate effect allows for faster and more accurate selection of viable sperm, which in turn improves fertilization and pregnancy outcome. ${ }^{5}$ The objective of this study was to compare ICSI cycle outcome when pentoxifylline or theophylline was used for identification of viable sperm in samples with all immotile sperm.

\section{Materials and methods}

This study was conducted on 112 couples visiting our center for male factor infertility treatment. The study duration was from Feb 2016 to Feb 2017. All male partners had either non motile sperm in the ejaculate or needed a surgical procedure to retrieve sperm. Pentoxifylline or theophylline was used to initiate sperm motility. During the study period pentoxifylline was used on 34 and theophylline on 78 samples. 


\section{Collection and Preparation of Semen Samples}

The sources of sperm in this study were normal ejaculates or sperm retrieved by surgical procedures namely fine needle aspiration (FNA), microsurgical epididymal sperm aspiration (MESA), microsurgical testicular sperm extraction (Micro-TESE) and testicular sperm extraction (TESE). Figure 1 shows different sites of taking the sperm or tissue samples.

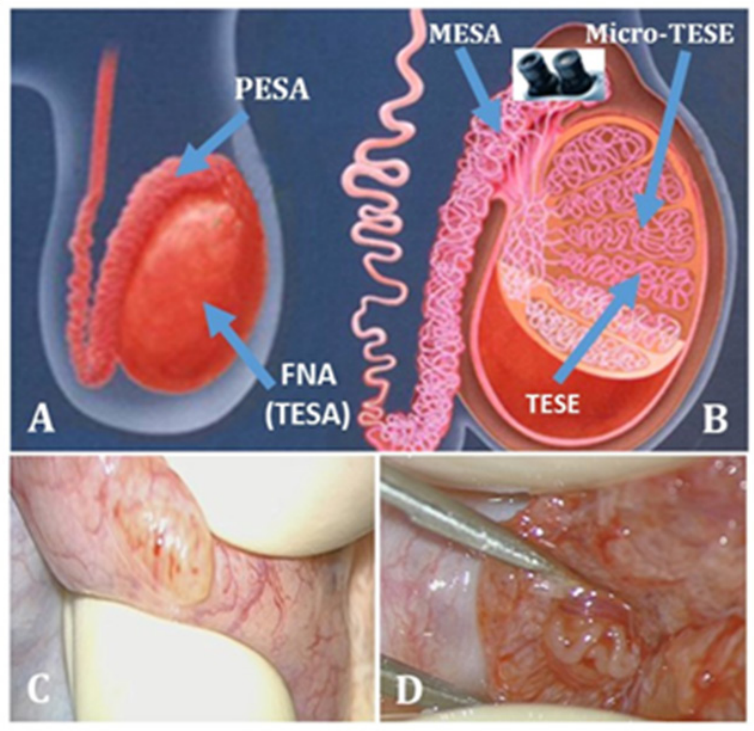

Figure I Different sites from where sperm or tissue samples were collected. $A$ and $B$ are graphical, however, $C$ and $D$ are real images: In case of $A$ and $C$, the samples were taken without and in $B$ and $D$ with the help of microscope.

The number of samples obtained from each sperm source for pentoxifylline and theophylline are given in Table 1. The normal ejaculates were collected by masterbation and examined after complete liquefaction. All these ejaculates had very rare immotile sperm. Each ejaculate was diluted with equal amount of sperm wash medium (V SPERM WASH, VitroMed, Germany), mixed gently and centrifuged at $1500 \mathrm{rpm}$ for 10 minutes. The supernatant was removed, pellet re-suspended to $100 \mu \mathrm{L}$ and kept at $23^{\circ} \mathrm{C}$ until the preparation of intracytoplasmic sperm injection dish (ICSI) dish preparation. All surgical procedures were performed in the operation room attached to the Andrology laboratory. The choice of procedure was based on patient's infertility history and decision of the surgeon. Majority of the ejaculates and FNA samples were used fresh as these were collected on the day of oocyte retrieval. However, majority of the Micro-TESE samples were retrieved well before oocyte retrieval and had to be used frozen-thawed.

Table I Sources of Sperm for ICSI in Pentoxifylline and Theophylline Group

\begin{tabular}{lllll}
\hline & \multicolumn{2}{l}{ Pentoxifylline } & Theophylline \\
\hline Sperm source & Fresh & Frozen-thawed & Fresh & Frozen-thawed \\
Ejaculate & 3 & 0 & 5 & $\mathrm{I}$ \\
FNA & 8 & 0 & 14 & 4 \\
MESA & 0 & 2 & 0 & 9 \\
Micro-TESE & $\mathrm{I}$ & $\mathrm{I}$ & 2 & 42 \\
TESE & $\mathrm{I}$ & 0 & 0 & $\mathrm{I}$ \\
\hline
\end{tabular}

\section{Ovarian stimulation and egg collection}

Ovarian stimulation was performed using a recombinant gonadotropin (Gonal-F, Merk, Serono) and GnRH antagonist protocol (Cetrotide, Merck Serono, Geneva, Switzerland). When the dominant follicle reached a diameter of $18 \mathrm{~mm}$ or greater, a single subcutaneous injection of 225 IU recombinant human chorionic gonadotropin (hCG) (Ovidrel, Merck Serono) was administered. Ultrasound-guided oocyte retrieval was performed 34-36 hours post hCG injection. All oocyte and embryo handling procedures were conducted on warm stages using standard laboratory methods.

\section{Preparation of ICSI dish with Theophylline or Pentoxifylline}

In this study, commercially available solution of theophylline (GM501 SpermMobil, GYNEMED, Germany) was used. Pentoxifylline solution at concentration of $5 \mathrm{mM}$ was prepared from powder supplied by Sigma (Cat \# P1784). The ICSI dish was prepared as shown in Figure 2. The ICSI drops were $5 \mu \mathrm{L}$, the media drop containing immotile sperm to which theophylline solution was added was $40 \mu \mathrm{L}$ and the media drop where sperm was rinsed after exposure to theophylline/pentoxifylline was $10 \mu \mathrm{L}$. The theophylline or pentoxifylline solution was warmed to $37^{\circ} \mathrm{C}$ in a non- $\mathrm{CO} 2$ incubator.

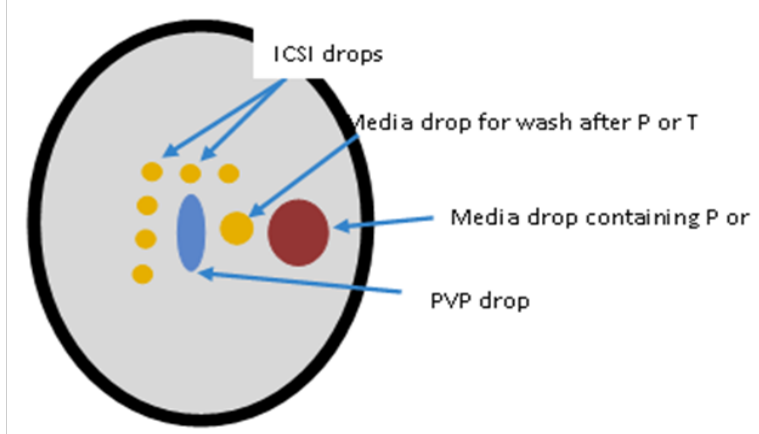

Figure 2 ICSI dish showing arrangement of pentoxifylline $(P)$ or theophylline (T), PVP and Media drops.

To facilitate sperm activation, $2 \mu \mathrm{L}$ solution of theophylline and 5 $\mu \mathrm{L}$ of $5 \mathrm{mM}$ pentoxifylline solution was added to the sperm containing drop. In cases with high number of retrieved oocytes, multiple $40 \mu \mathrm{L}$ drops were made and theophylline or Pentoxifylline was added to each drop to get more viable sperm. The ICSI dish was then placed on a warm plate at $37^{\circ} \mathrm{C}$ for $10 \mathrm{~min}$ before search for viable sperm for ICSI.

The viable sperm showing visible movement were picked, rinsed in media drop, immobilized in PVP drop and injected into the egg. In case of non-availability of motile sperm, eggs were injected with immotile sperm having flexible tail. In case of very poor sperm acrosome or globozoospermia, artificial oocyte activation was performed by GM508 Cult-Active (GYNEMED, Denmark). The remaining procedures like oocyte denudation, ICSI, embryo culture, fertilization check, embryo development check and embryo transfer were performed per our standard laboratory protocols.

\section{Statistical analysis}

Student T-test and Chi-square test were used to compare data. Results were presented as means \pm standard deviation (SD). Statistical significance was considered at a $\mathrm{p}<0.05$.

\section{Results}

Pentoxifylline was used in 34 cases and it activated sperm motility in $88 \%$ cases. Theophylline was used in 78 cases and it activated 
motility in $90 \%$ cases. All remaining cases, in which no motile sperm was observed after exposure to theophylline or pentoxifylline, were frozen-thawed samples, and no or very poor fertilization was observed. Ages of men and women, number of eggs retrieved and number of mature oocytes were similar in both groups $(\mathrm{P}<0.05)$ (Table 2$)$.

Number of eggs injected, number fertilized and number of embryos transferred were similar in both groups $(\mathrm{P}<0.05)$. Embryo transfer was performed based on our clinic's standard policy either on day 3 or day-5. On day-3, a maximum of 2 embryos were transferred in less than $35 \mathrm{yr}$ and 3 embryos in 35-39 yr old patients (Table 3). The ratio of day 3 to day 5 transfers was similar in both groups. In pentoxifylline treated group $38 \%$ and in theophylline treated group $40 \%$ transfers were performed on day-5.

Table 2 Number of patients, their ages, oocytes retrieved and mature oocytes in both groups.

\begin{tabular}{llllll}
\hline Treatment & Patients & Age male (Mean $\mathbf{4 S D})$ & age female (Mean $\mathbf{4 S D})$ & \# Oocytes retrieved \# Mature oocytes \\
\hline Pentoxyphylline & 34 & $38.3 \pm \mathrm{II}$ & $32.2 \pm 6.4$ & $13 \pm 8$ & $9 \pm 7$ \\
Theophyssine & 78 & $39.2 \pm 10.7$ & $31.5 \pm 6.1$ & $1 \mathrm{I} \pm 6$ & $8 \pm 5$ \\
Over All & $\mathrm{II} 2$ & $38.9 \pm 10.7$ & $31.7 \pm 6.2$ & $12 \pm 7$ & $8 \pm 6$ \\
\hline
\end{tabular}

Values are Mean $\pm \mathrm{SD}$.

Table 3 Number of oocytes injected, number fertilized and number of embryos transferred in pentoxifylline and theophylline treated cases

\begin{tabular}{llll}
\hline & \# ICSI (Mean \pm SD) & \# Fert (Mean \pm SD) & \# ET (Mean \pm SD) \\
\hline Pentoxyphylline & $9.0 \pm 6.6$ & $4.5 \pm 3.6$ & $2.0 \pm 0.7$ \\
Theophyssine & $8.4 \pm 5.0$ & $3.6 \pm 2.7$ & $2.0 \pm 0.7$ \\
Over all & $8.6 \pm 5.6$ & $3.9 \pm 3.0$ & $2.0 \pm 0.7$ \\
\hline
\end{tabular}

Values are Mean \pm SD. $P<0.05$.

Biochemical and clinical pregnancies were also similar in both groups. Biochemical pregnancy rate was $32.4 \%$ and $33 \%$ and clinical pregnancy rate was $21 \%$ and $22 \%$ in pentoxifylline and theophylline groups, respectively (Figure 3). The fertilization rate was in pentoxifylline treated cases was $57 \%$ and in theophylline $45 \%$. This difference was statistically non-significant $(\mathrm{P}<0.05)$. The miscarriage rate of $9 \%$ in pentoxifylline treated cases was significantly higher than $1.2 \%$ in theophylline treated cases $(\mathrm{P}>0.05)$. So far 11 deliveries of normal healthy babies have been reported; 2 from pentoxifylline and 9 from theophylline.

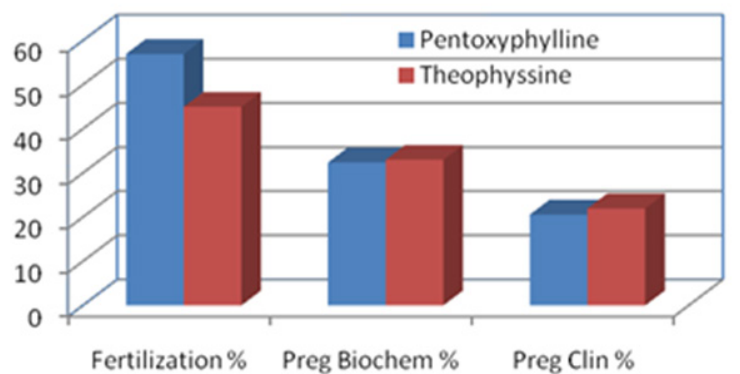

Figure 3 Cycle outcome in pentoxifylline and theophylline treated sperm. PregBiochem = Rise in $\beta$ hCG in blood;

Preg Clinic $=$ Observation of heartbeat and fetal sac by ultrasound.

\section{Discussion}

Out of 112 patients, only 3 produced sperm by masturbation and had all sperm non-motile. For all other cases, the sperm were retrieved by a surgical procedure. Regardless of the fresh or frozen status of the sample, all sperm were immotile before or after processing. Both motility activating agents; pentoxifylline and theophylline were effective in about $90 \%$ cases, however, a bare minimal motility was observed after exposure to the agent. The exposure, however, facilitated ICSI procedure as less time was needed to find viable sperm. Both activating agents are methylxanthine derivatives. Both induce sperm motility by inhibiting phosphodiesterase activity thus increasing intracellular cAMP levels. ${ }^{7}$ Pentoxifylline significantly increases creatine kinase enzyme activity of spermatozoa. This enzyme is indispensable for sperm function because it catalyses the regeneration of ATP from the chemical shuttle between creatine and creatine phosphate. ${ }^{12}$

Slightly more percent fertilization was observed in cases treated with pentoxifylline (Figure 2), however, this difference was statistically non-significant $(\mathrm{P}<0.05)$ and this difference did not result in higher biochemical or clinical pregnancy rate. In earlier studies, pentoxifylline has been shown to significantly improve fertilization rates and the number of available embryos as comparison to control but these improvements were not reflected in the corresponding increase in clinical pregnancy. ${ }^{7}$

We observed $9 \%$ miscarriages in the group treated by Pentoxifylline. It is higher than that observed in theophylline treated cases. A previous report observed significant increase in sperm DNA damage following application of pentoxifylline to samples from individuals with different sperm parameters. ${ }^{10}$ Although it has been widely used for stimulating motility, its use is not universally accepted for its possible detrimental effects on oocytes, embryos or newborns. Some investigators did not find any increase of adverse outcomes, ${ }^{13}$ including malformation rates. ${ }^{2}$ It has been suggested to avoid prolonged contact of xanthine derivatives with embryos since it might result in marked morphological changes. Data from animal studies show that prolonged incubation of mouse embryos led to developmental retardation or embryo death. ${ }^{5}$ We washed the selected spermatozoa in pentoxifylline or theophylline free media drop, transferred to PVP for immobilization and then injected into oocyte, thus reducing the risk of a biological hazard on oocytes. Further, theophylline is available in a standardized commercial product. This ready-to-use stock solution minimizes preparation variation.

Several options are now available to embryologists for detection of viable sperm for ICSI from samples with all immotile sperm.

i. Use of sperm motility activating agents like Pentoxifylline, ${ }^{4}$ theophylline, ${ }^{5}$ papaverine ${ }^{6}$ and platelet activating factor. ${ }^{10}$

ii. A reasonable approach is to use the ICSI pipette to test the elasticity of the sperm tail. However, there is no guarantee that sperm with elastic tail has viable nucleus. 
iii. Another commonly used method is HOS test. This test determines functionality of sperm plasma membrane. If viable sperm are incubated in a hypo-osmotic solution, the tail tip coils. ${ }^{3} \mathrm{~A}$ practical method to make hypo-osmotic solution is to mix sperm wash media with sterile deionized water in 1:1 ratio.

iv. A single shot of diode laser on the tip of the sperm tail cause curling of the tail. ${ }^{14}$ This test is also based on the functional integrity of sperm tail membrane. The most reliable strategy is partial restoration of sperm motility by use of motility activating agent.

\section{Conclusion}

Results of this study indicate that theophylline is safe in identifying viable sperm for ICSI in cases with all non-motile sperm in fresh or frozen semen samples or those retrieved by surgical procedure. In this study theophylline and pentoxifylline were not effective in about $10 \%$ cases. There is a need to develop a method which is easily applicable, specific, allows fast identification of best sperm and causes no harm to egg or spermatozoa.

\section{Acknowledgments}

None.

\section{Conflicts of interest}

None.

\section{References}

1. Montjean D, Courageot J, Altié A, et al. Normal live birth after vitrified warmed oocytes intracytoplasmic sperm injection with immotile spermatozoa in a patient with Kartagener's syndrome. Andrologia. 2015;47(7):839-845.

2. Navas P, Paffoni A, Intra G, et al. Obstetric and neo-natal outcomes of ICSI cycles using pentoxifylline to identify viable spermatozoa in patients with immotile spermatozoa. Reprod Biomed Online. 2017;34(4):414-421.

3. Mangoli V, Mangoli R, Dandekar S, et al. Selection of viable spermatozoa from testicular biopsies: a comparative study between pentoxifylline and hypoosmotic swelling test. Fertil Steril. 2011;95(2):631-634
4. Yildirim G, Ficicioglu C, Akcin O, et al. Can pentoxifylline improve the sperm motion and ICSI success in the primary ciliary dyskinesia? Arch Gynecol Obstet. 2009;279(2):213-215.

5. Ebner T, Tews G, Mayer RB, et al. Pharmacological stimulation of sperm motility in frozen and thawed testicular sperm using the dimethylxanthine theophylline. Fertil Steril. 2011;96(6):1331-1336.

6. Terriou P, Hans E, Cortvrindt R, Avon C, Charles O, et al. (2015) Papaverine as a replacement for pentoxifylline to select thawed testicular or epididymal spermatozoa before ICSI. Gynecol Obstet Fertil 43(12): 786-790.

7. Nordhoff V. How to select immotile but viable spermatozoa on the day of intracytoplasmic sperm injection? An embryologist's view. Andrology. 2014;3(2):156-162.

8. Nordhoff V, Schüring AN, Krallmann C, et al. Optimizing TESE-ICSI by laser-assisted selection of immotile spermatozoa and polarization microscopy for selection of oocytes. Andrology. 2013;1(1):67-74.

9. Charehjooy N, Najafi MH, Tavalaee M, et al. Selection of Sperm Based on Hypo-Osmotic Swelling May Improve ICSI Outcome: A Preliminary Prospective Clinical Trial. Int J Fertil Steril. 2014;8(1):21-28.

10. Unsal E, Turan V, Aktuna S, et al. Effects of pentoxifylline and platele activating factor on sperm DNA damage. Eur J Obstet Gynecol Reprod Biol. 2016;197:125-129.

11. Wang R, Sikka SC, Veeraragavan K, et al. Platelet activating factor and pentoxifylline as human sperm cryoprotectants. Fertil Steril. 1993;60(4):711-715.

12. Banihani SA, Abu-Alhayjaa RF. The activity of seminal creatine kinase is increased in the presence of pentoxifylline. Andrologia. 2016;48(5):603-604.

13. Amer M, Metawae B, Hosny $\mathrm{H}$, et al. Beneficial effect of adding Pentoxifylline to processed semen samples on ICSI outcome in infertile males with mild and moderate asthenozoospermia: A randomized controlled prospective crossover study. Iran J Reprod Med. 2013;11(11):939-944.

14. Aktan TM, Montag M, Duman S, et al. Use of a laser to detect viable bu immotile spermatozoa. Andrologia. 200436(6):366-369. 